J. Sustain. Wireless Syst. (2019)

Vol.01/ No. 04

Pages: 225-234

http://irojournals.com/irosws/

DOI: https://doi.org/10.36548/jsws.2019.4.003

\title{
COMPUTATIONAL OFFLOADING FOR PERFORMANCE IMPROVEMENT AND ENERGY SAVING IN MOBILE DEVICES
}

\author{
Dr. Joy Iong Zong Chen, \\ Professor, Department of Electrical Engineering, \\ Da-Yeh University, \\ Taiwan. \\ Email id: jchen@mail.dyu.edu.tw
}

\begin{abstract}
The mobiles devices such as the smart phones and the wearables take a vital role in our daily life scenario as they are been used as alternative for many devices apart from communication. The mobile devices that are controlled in terms of dimensions and weightiness to make them easy and flexible for handling in turn limits the computational energy, storage and the lifetime of the battery. So this entails the need for the external device to support the mobile devices by providing a computational power, storage and energy, this is known as the computational offloading. So the paper puts forth the mobile cloud services as an external platform to offload the resource intensive computation tasks of the mobile devices to enhance the performance of the mobile devices in terms of storage, energy consumption and battery life. The performance evaluation of the mobile cloud based offloading in the mobile devices proves the efficiency of the proffered method in terms of storage, energy and battery lifetime.
\end{abstract}

Keywords: Computational Offloading, Mobile Devices, Performance Improvement, Energy Saving, Storage Enhancement and Extended Battery Life

\section{INTRODUCTION}

As the mobile devices are becoming more and more predominant among wide range of users it has become ubiquitous. The capability of the mobile devices to support and execute applications with diverse requirements that range from simple mathematical to complex system remains as the reason for their ubiquitousness [1]. The application used in the mobile devices overflow the device limitations in terms of the computation, energy and the data resources paving way for the necessity of the offloading strategies for the mobile devices [2]. The fig.1 below shows the offloading architectures for the devices with the mobile nature.

ISSN: 2582-3167 (online) 
J. Sustain. Wireless Syst. (2019)

Vol.01/ No. 04

Pages: 225-234

http://irojournals.com/irosws/

DOI: https://doi.org/10.36548/jsws.2019.4.003

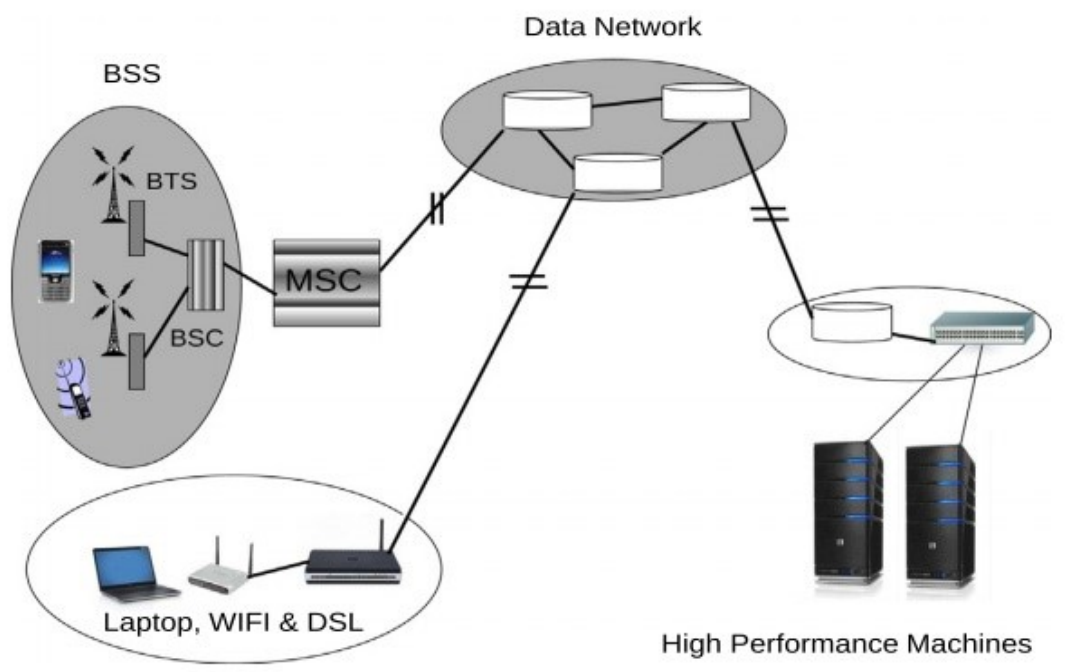

Fig.1 Architecture for Offloading [1]

The offloading scheme helps out in bring down heavy resource utilization in the portable devices that are incorporated with the poor resources such as the limited processor performance, storage capacity, network bandwidth, and battery life [3].

The paper put forth the mobile cloud service that is framed using the nearby available mobile devices for enabling the offloading for the mobile device that finds it hard to handle the resource and the computational intensive applications.

The paper is organized with the 2. Related works, 3. Proposed work, 4. Performance analysis and 5. Conclusion.

\section{RELATED WORKS}

Khan et al [2], the" comprehensive survey based on the necessity and the offloading strategies to resource constrained mobile devices, that require intensive computations to be offloaded in order to bring down the issue in the energy consumption and the execution time".

ISSN: 2582-3167 (online) 
J. Sustain. Wireless Syst. (2019)

Vol.01/ No. 04

Pages: 225-234

http://irojournals.com/irosws/

DOI: https://doi.org/10.36548/jsws.2019.4.003

Mtibaa, et al [1] the paper "highlights the various offloading algorithms for the mobile device clouds and utilizes the various platforms to execute the offloading experiments using the MDC platform, and also utilizes the offloading selection issues in the mobile device by putting forth a various social based algorithms.

Kumar, et al [3], the author puts forth "the survey paper that presents the over view of the back ground techniques, systems and the research areas that are related to the offloading strategies". The fig.2 below shows the offloading technologies

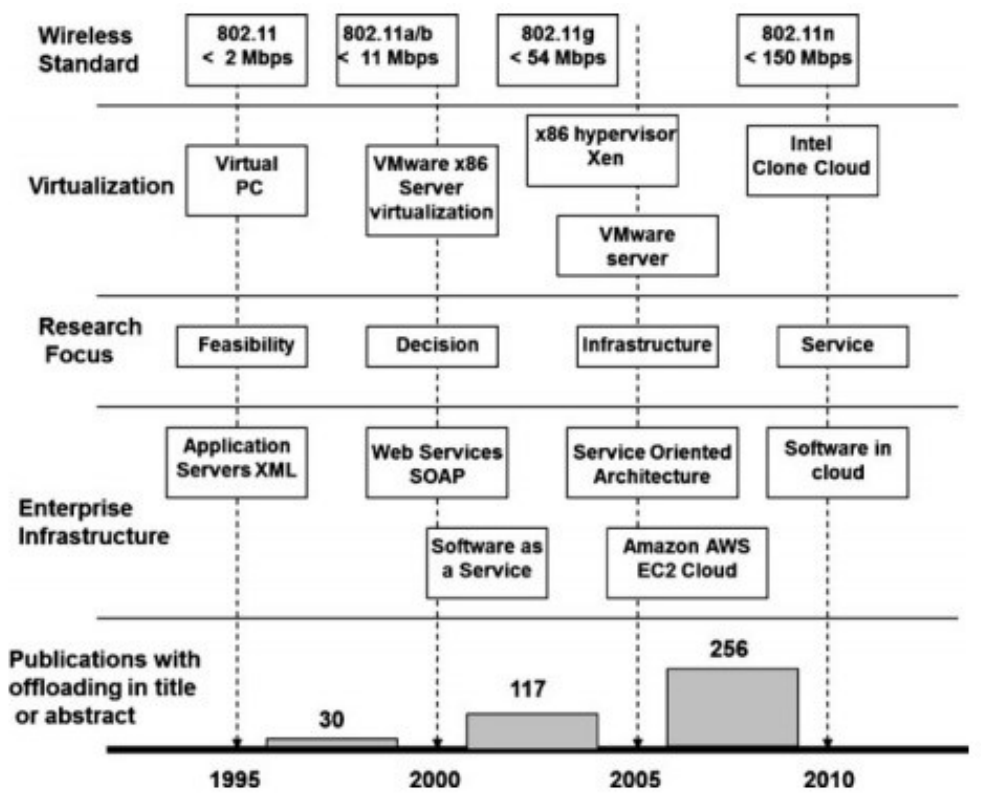

Fig. 2 Technologies Used in Offloading [3]

Fahim et al [4], the paper initially highlights the gain in the computational time and the energy usage that could be achieved using the offloading strategies. The paper initially proceeds with the offloading tasks to the nearby device in the mobile cloud and utilizes the test bed which consists of four android devices along with the energy measurement technique to validate the suspects of the author in terms of the gain in time and gain in energy.

Kovachev, et al [5], the author presents an adaptive computational offloading for the computationally intensive applications that are too complex to be handled by the mobile devices that are limited in terms of the storage capacity, battery usage and the energy consumption.

ISSN: 2582-3167 (online) 
J. Sustain. Wireless Syst. (2019)

Vol.01/ No. 04

Pages: 225-234

http://irojournals.com/irosws/

DOI: https://doi.org/10.36548/jsws.2019.4.003

Kumar et al [6], the article is about the benefits of the computational offloading for the mobile devices in terms of the energy, the figure. 3 below shows when an offloading technique would be beneficial. The figure. 3 stress that the offloading would be beneficial on large amount of computational instructions and minimized amount of communication data bytes.

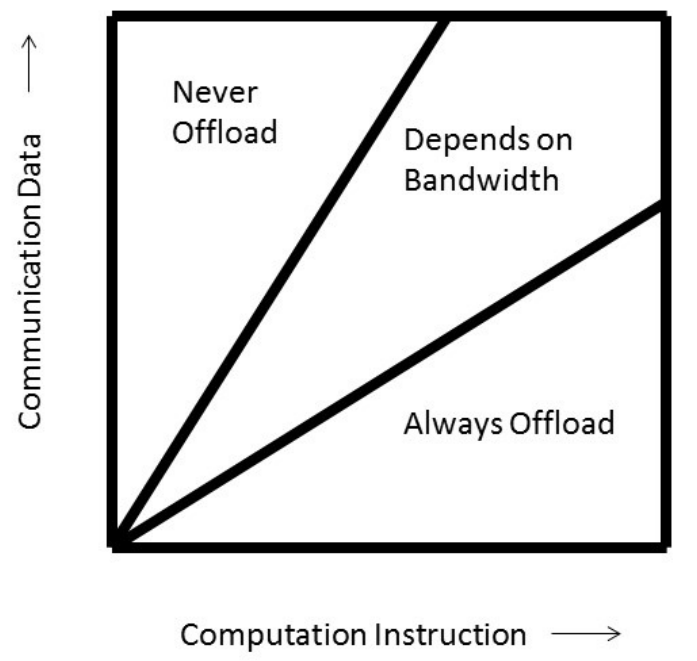

Fig. 3 Necessity of Offloading [6]

Jiao et al [7], "the cloud based computational offloading for the mobile devices is presented in the paper by investigating the state of art code offloading for the mobile devices and highlighting the important challenges that encountered in developing an efficient cloud based offloading frame work along with the necessary technologies utilized in facilitating the implementation of the frame work".

Mao et al [8], the author "investigates the green mobile edge computing system with the energy harvesting devices to develop an effective computational offloading strategy. Utilizing the Lyapunov optimization based - computation offloading algorithm that jointly decides the offloading decision, CPU cycle frequencies associated with the mobile execution and the transmission power for the computational offloading".

Shi, et al [9] the paper presents the design and the implementation of the COSMOS (computational offloading as service for mobile devices) that bridges the gap between the providing of the service and its efficient management to both improve the offloading performance and minimize the monetary cost"

ISSN: 2582-3167 (online) 
J. Sustain. Wireless Syst. (2019)

Vol.01/ No. 04

Pages: 225-234

http://irojournals.com/irosws/

DOI: https://doi.org/10.36548/jsws.2019.4.003

Zhang, et al [10], to enhance the capabilities of the mobile devices that are resource constrained the paper puts forward the elastic application model to ensure a seamless and a transparent use of the cloud resources to enlarge the capabilities of the mobile devices especially with limited resource availabilities.

Qian et al [11], "the performance of the mobile devices depends on the longevity of the battery life; the author puts forth the offloading of the computations to the resourceful server to enhance the performance of the mobile applications. The author utilizes the JADE to monitor the device application status and automatically decides the whether the application has to be offloaded or not".

Chen et al [12] the paper presents the extended client server collaboration by offloading few computations such as the execution method and the dynamic compilation by utilizing the object serialization feature of java. Olsson, et al [13] PSO optimization technique and applications are discussed in the paper. Qi, et al [14] the user based k means algorithm is utilized in offloading for the heterogeneous type network.

\section{OFFLOADING FOR MOBILE DEVICES}

The offloading for the mobile devices in this paper is planned using the mobile cloud services, that is the mobile devices nearby with the capacity to extend support for the computational intensive task are framed as an network in the adhoc manner, by identifying the resourceful devices to act as the external platform to support the mobile application that are computational and resource intensive. The fig. 4 below shows the flow diagram that includes the various phases in the proposed process of offloading for the mobile devices.

ISSN: 2582-3167 (online) 
J. Sustain. Wireless Syst. (2019)

Vol.01/ No. 04

Pages: 225-234

http://irojournals.com/irosws/

DOI: https://doi.org/10.36548/jsws.2019.4.003

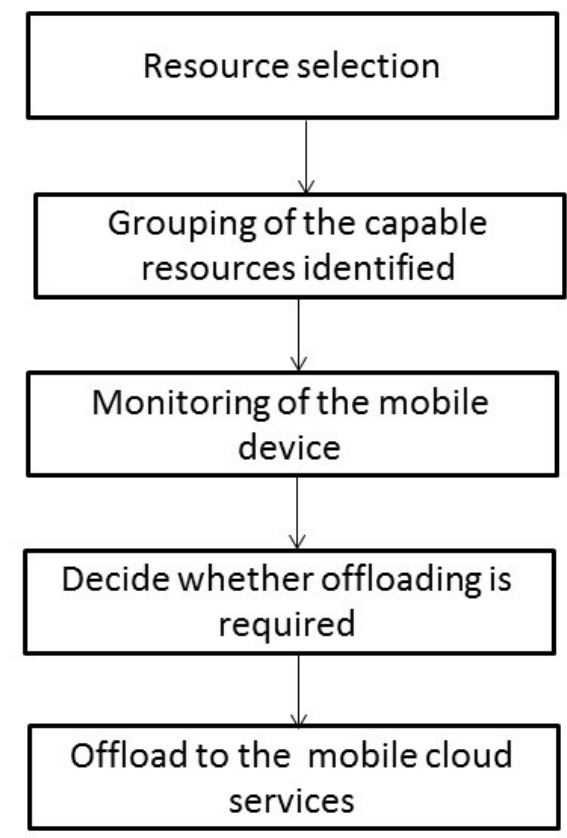

Fig.4 Phase in the Proposed Process

The proposed method initiates with the resource selection process by gathering the information of the nearby volunteering devices by sending a greeting message and determines the resource capacity of the devices by enumerating the available cloud storage space, battery life and the processing capacity by applying the particle swarm optimization [13]. Once the resourceful devices are selected, the user based kmeans [14] is applied to group the devices with the best resources. In the meantime the JADE [11] is used in monitoring the status of the mobile devices that requires an off-loading of an application and deciding whether the offloading is required. Once the mobile device necessitates an offloading, the adaptive method [5] of offloading is used to offload the mobile application to the mobile cloud services. The algorithm given below in the table .1 provides the description of the phases involved in the offloading.

ISSN: 2582-3167 (online) 
J. Sustain. Wireless Syst. (2019)

Vol.01/ No. 04

Pages: 225-234

http://irojournals.com/irosws/

DOI: https://doi.org/10.36548/jsws.2019.4.003

\begin{tabular}{|c|c|}
\hline $\begin{array}{l}\text { PHASES IN THE OFF- } \\
\text { LOADING }\end{array}$ & ALGORITHM \\
\hline \multirow{4}{*}{ Resources Selection } & $\begin{array}{l}\text { Generate greeting to gather the information of the voluntering } \\
\text { devices nearby }\end{array}$ \\
\hline & $\begin{array}{l}\text { The reply is generated form volunteering devices along with the } \\
\text { information of the processing capacity }(P C) \text {, storage availability } \\
\text { (Sa) and the Residual energy of the battery }(B L)\end{array}$ \\
\hline & $\begin{array}{l}\text { Apply particle swarm optimization to enumerate the devices } \\
\text { with the } \max \{P c, S a, B I\}\end{array}$ \\
\hline & $\begin{array}{l}\text { Arrange the devices according to the enumerated capacities in } \\
\text { ascending order }\end{array}$ \\
\hline \multirow{2}{*}{$\begin{array}{l}\text { Clustering the Resourceful } \\
\text { Devices }\end{array}$} & $\begin{array}{l}\text { The output of the PSO being fed to the next stage of clustering } \\
\text { to group the resourceful devices identified. }\end{array}$ \\
\hline & $\begin{array}{l}\text { The identified results with the similar }\{P c, S a, B l\} \text { are } \\
\text { grouped applying the } \mathrm{K} \text { means algorithm. }\end{array}$ \\
\hline \multirow{2}{*}{$\begin{array}{l}\text { Monitoring of the mobile } \\
\text { device that needs } \\
\text { offloading }\end{array}$} & $\begin{array}{c}\text { The mobile device with the limited resource availabilities is } \\
\text { monitored using the JADE }\end{array}$ \\
\hline & $\begin{array}{l}\text { The JADE monitors the status of the mobile and its capability to } \\
\text { handle the application }\end{array}$ \\
\hline \multirow[b]{2}{*}{ Decision on off-loading } & $\begin{array}{l}\text { Determine the Mobile Status (Ms) for the mobile device based } \\
\text { on the }\{P c, S a, B l\}\end{array}$ \\
\hline & $\begin{array}{l}\text { If } M S=\{P c, S a, B l\}<\text { then the verge level and the } \\
\text { computational instructions are higher and the communication } \\
\text { bytes are low the offloading takes places }\end{array}$ \\
\hline \multirow{3}{*}{$\begin{array}{l}\text { Off-loading to Mobile } \\
\text { Cloud Services }\end{array}$} & $\begin{array}{l}\text { The cost function }\left(C_{f}\right) \text { for the offloading is enumerated based } \\
\text { on the memory cost }\left(C_{m}\right) \text {, cost of the transfer }\left(C_{t}\right) \text { and the cost } \\
\text { of the processing }\left(C_{c p u}\right) \text {. }\end{array}$ \\
\hline & $\begin{array}{l}C_{f}=C_{t}+C_{m n}+C_{c p n} \text {, and further determines also the energy } \\
\text { cost }\left(E_{c}\right) \text { and the execution time }\left(E_{t}\right) \text {. }\end{array}$ \\
\hline & $\begin{array}{l}\text { The mobile cloud with the } \min \left\{C_{f},\left(E_{c}, E_{t}\right\} \text { is selected for }\right. \\
\text { offloading from the clustered groups of mobile devices. }\end{array}$ \\
\hline
\end{tabular}

Table.1 Algorithm for the Proposed Off-loading

\section{Performance Analysis}

The evaluation of the proposed process of offloading for the mobile device that are computational and resource intensive using the network simulator-2 shows enhancement in the performance of the mobile devices on the grounds of the storage, energy consumption and battery longevity. The fig. 5 shows the energy consumption of the mobile devices when offloaded with the mobile cloud and the other conventional services. 
J. Sustain. Wireless Syst. (2019)

Vol.01/ No. 04

Pages: 225-234

http://irojournals.com/irosws/

DOI: https://doi.org/10.36548/jsws.2019.4.003

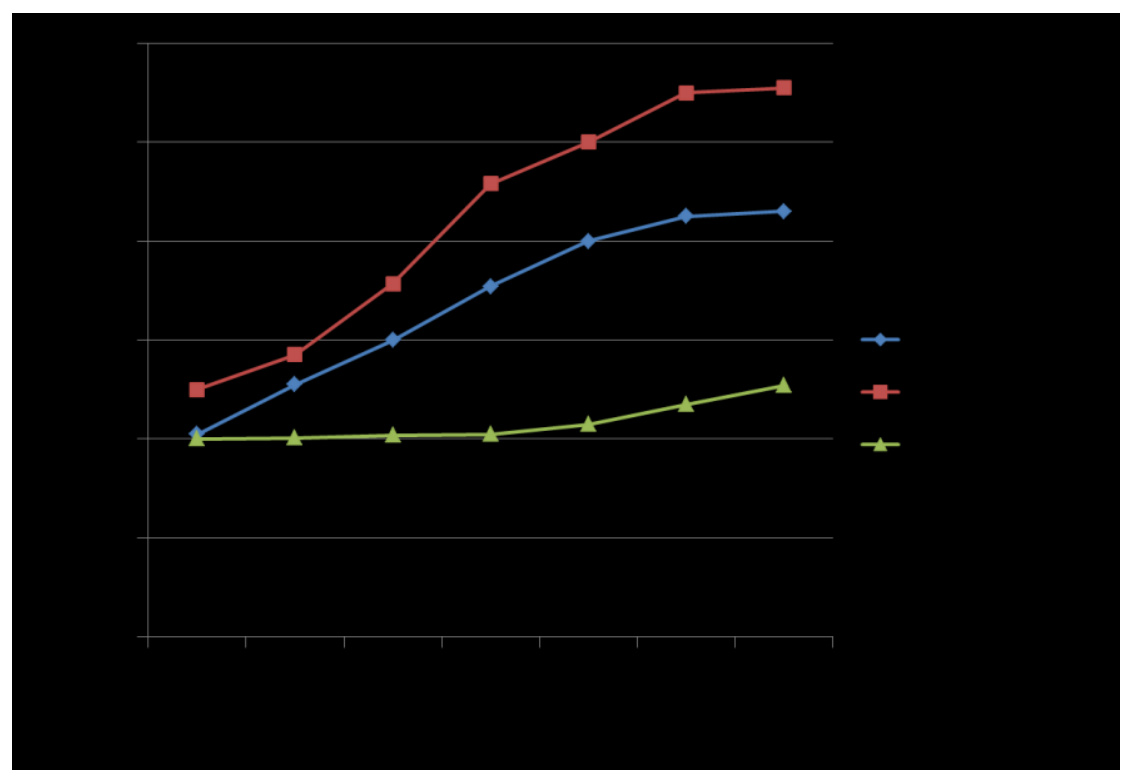

Fig. 5 Energy Consumption

The fig. 6 shows the percentage of the storage availability and the battery longevity of the mobile devices when offloaded to the mobile cloud services for applications of different sizes under different scenarios.

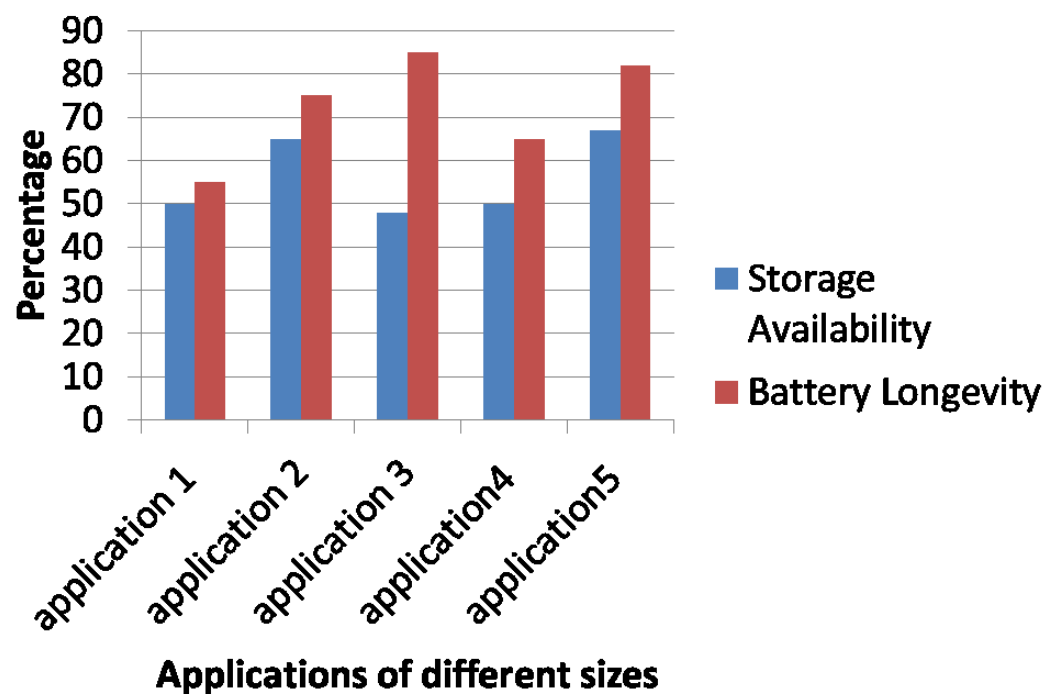

Fig.6 Enhancement in storage and Battery Longevity 
J. Sustain. Wireless Syst. (2019)

Vol.01/ No. 04

Pages: 225-234

http://irojournals.com/irosws/

DOI: https://doi.org/10.36548/jsws.2019.4.003

The paper provides an efficient way of off-loading to improvise the performance of the mobile devices. Further the method to protect the information's offloaded from being misused is continued in its future work.

\section{CONCLUSION}

The computational offloading which is efficient method of transferring the resource intensive computational task to the external platform, to improvise the processor in terms of the computational power, storage and the battery life time is presented in the paper for the mobile devices such as the smart wearables and the smart phones. The paper utilizes the cloud services as the external platforms for the portable devices to offload its computational and the resource intensive tasks to the cloud services to minimize the battery usage, energy and the storage consumption of the mobile devices. The performance improvement of the mobile devices using the cloud based offloading is evaluated using the network simulator-2 to evince the efficiency of the system. Further the paper is to continue with the over view of the security issues related to the computational offloading and the solutions available for them.

\section{References}

[1] Khan, Minhaj Ahmad. "A survey of computation offloading strategies for performance improvement of applications running on mobile devices." Journal of Network and Computer Applications 56 (2015): $28-40$.

[2] Mtibaa, Abderrahmen, Khaled A. Harras, and Afnan Fahim. "Towards computational offloading in mobile device clouds." In 2013 IEEE 5th international conference on cloud computing technology and science, vol. 1, pp. 331-338. IEEE, 2013.

[3] Kumar, Karthik, Jibang Liu, Yung-Hsiang Lu, and Bharat Bhargava. "A survey of computation offloading for mobile systems." Mobile Networks and Applications 18, no. 1 (2013): 129-140.

[4] Fahim, Afnan, Abderrahmen Mtibaa, and Khaled A. Harras. "Making the case for computational offloading in mobile device clouds." In Proceedings of the 19th annual international conference on Mobile computing \& networking, pp. 203-205. ACM, 2013.

[5] Kovachev, Dejan, Tian Yu, and Ralf Klamma. "Adaptive computation offloading from mobile devices into the cloud." In 2012 IEEE 10th International Symposium on Parallel and Distributed Processing with Applications, pp. 784-791. IEEE, 2012.

[6] Kumar, Karthik, and Yung-Hsiang Lu. "Cloud computing for mobile users: Can offloading computation save energy?." Computer 4 (2010): 51-56. 
J. Sustain. Wireless Syst. (2019)

Vol.01/ No. 04

Pages: 225-234

http://irojournals.com/irosws/

DOI: https://doi.org/10.36548/jsws.2019.4.003

[7] Jiao, Lei, Roy Friedman, Xiaoming Fu, Stefano Secci, Zbigniew Smoreda, and Hannes Tschofenig. "Cloud-based computation offloading for mobile devices: State of the art, challenges and opportunities." In 2013 Future Network \& Mobile Summit, pp. 1-11. IEEE, 2013.

[8] Mao, Yuyi, Jun Zhang, and Khaled B. Letaief. "Dynamic computation offloading for mobile-edge computing with energy harvesting devices." IEEE Journal on Selected Areas in Communications 34, no. 12 (2016): 3590-3605.

[9] Shi, Cong, Karim Habak, Pranesh Pandurangan, Mostafa Ammar, Mayur Naik, and Ellen Zegura. "Cosmos: computation offloading as a service for mobile devices." In Proceedings of the 15th ACM international symposium on Mobile ad hoc networking and computing, pp. 287-296. ACM, 2014.

[10] Zhang, Xinwen, Anugeetha Kunjithapatham, Sangoh Jeong, and Simon Gibbs. "Towards an elastic application model for augmenting the computing capabilities of mobile devices with cloud computing." Mobile Networks and Applications 16, no. 3 (2011): 270-284.

[11] Qian, Hao, and Daniel Andresen. "Extending mobile device's battery life by offloading computation to cloud." In Proceedings of the Second ACM International Conference on Mobile Software Engineering and Systems, pp. 150-151. IEEE Press, 2015.

[12] Chen, Guangyu, B-T. Kang, Mahmut Kandemir, Narayanan Vijaykrishnan, Mary Jane Irwin, and Rajarathnam Chandramouli. "Studying energy tradeoffs in offloading computation/compilation in javaenabled mobile devices." IEEE Transactions on Parallel and Distributed Systems 15, no. 9 (2004): 795-809.

[13] Olsson, Andrea E. Particle swarm optimization: theory, techniques and applications. Nova Science Publishers, Inc., 2010.

[14] Qi, Weijie, Baoling Zhang, Bozhong Chen, and Jie Zhang. "A user-based K-means clustering offloading algorithm for heterogeneous network." In 2018 IEEE 8th Annual Computing and Communication Workshop and Conference (CCWC), pp. 307-312. IEEE, 2018.

ISSN: 2582-3167 (online) 